\title{
A novel low-latency BICM system by quantizing the constellation on the demodulation end
}

\author{
Jianping $\mathrm{Li}^{1, \mathrm{a}}$, Yameng Shi ${ }^{2, b^{*}}$ \\ ${ }^{1,2}$ School of Information Engineering, Communication University of China, Beijing, China \\ ae-mail: turbo_code@163.com, be-mail:yayaya_12002@163.com
} Keywords: demodulation, quantification, table look-up, computation complexity, Additive White
Gaussian Noise

\begin{abstract}
This paper proposes a new demodulation structure by quantizing the constellation and using a lookup table. When the modulated noisy symbols are received by demodulation end, it's not to calculate the Euclidean distances between a symbol and every constellation points, but quantize each dimension of this symbol's coordinate value in the constellation, and check the bit soft values corresponding to this quantification point against a lookup table which is known by a pretreatment. The proposed method will omit a large number of the calculations for demodulation and simplifies the system. Employing look-up table instead of traditional computing can reduce the delay by $72.4 \%$ to $93.9 \%$ when the quantification interval is from 0.1 to 0.4 . Meanwhile, the performance gain only reduces about $0.1 \mathrm{~dB}$.
\end{abstract}

\section{Introduction}

Coded modulation (CM) [1] combines the coding and modulation for improved performance. Bit-interleaved coded modulation (BICM) [2, 3] brings a better performance by bit-wise interleaving at the encoder output over a Rayleigh fading channel. Some proposed methods optimize the demodulation part such as $[4,5]$.

Demodulator outputs the bit soft information of each code in order to get a lower bit-error-rate (BER) by adopting the soft decoding. Log Maximum A Posteriori (Log-Map) [6], Maximum LogMAP (Max-Log-Map) [7] and Soft-Output Viterbi (SOVA) [8] are the three decoding algorithms in common use. There are two steps in the process of traditional demodulation. First, calculate a matrix composed of Euclidean distances between a symbol that is received from the noisy channel and every constellation points; second, compute a sequence of bit soft values by this matrix adopting a demodulation algorithm, and take the sequence as a soft input of decoding part finally. The series of demodulation process above is tedious and time-consuming.

This paper presents a quantitative method for constellation graph to simplify the demodulation process. First, constellation graph is divided into small regions and the transmitted symbol from the noisy channel is quantized as a fixed point within the region where it is mapped; second, check the bit soft values of this fixed point against a lookup table which has already been calculated by putting every fixed point value into the traditional algorithm. Therefore, tabulation is not included in the entire communication process and it's just a pretreatment. The contributions of this paper can be summarized as follows: using simple and low-latency processes of quantification and table lookup replaces the complex and high-latency algorithms with decreasing a bit little performance. These theoretical results are supported in Section III by means of simulation and conclusions are presented in Section IV.

\section{System Model}

The proposed demodulation structure consists of three parts: pretreatment part, quantizing received symbols part and bit value search part.

Pretreatment. The purpose of pretreatment is to get the table for demodulation query. There are four steps to make a table before regular communications. 
Step1: calculate a maximum value "Q_MAX" as the boundary of the quantized area. First, produce a string of zero sequence " $0000 \ldots$.." and send the signal through the channel under test. Then, work out some numerical characteristics by doing statistical analysis and channel estimation with these noisy signals after repeated experiments.

An experiment with Additive White Gaussian Noise (AWGN) is employed as an experiment to analyze. The mean value " $a$ " and variance " $\sigma^{2}$ " which are the two numerical characteristics used to characterize Gaussian random process can be obtained by the statistical analysis, and the probability density distribution curve can be drawn.

Suppose it is a standard Gaussian distribution, i.e., $\mathrm{a}=0$, and $\sigma^{2}=1$. Fig. 1 shows this probability density distribution.

The probability density expression is

$$
f(x)=\frac{1}{\sqrt{2 \pi} \sigma} \exp \left(-\frac{(x-a)^{2}}{2 \sigma^{2}}\right)
$$

where $x$ presents a signal amplitude which has been disturbed by AWGN. The distribution expression is

$$
F(x)=P(\xi \leq x)=\int_{-\infty}^{x} \frac{1}{\sqrt{2 \pi} \sigma} \exp \left[-\frac{(z-a)^{2}}{2 \sigma^{2}}\right] d z
$$

It can be solved that $\mathrm{F}(\mathrm{x})=0.9987$ when $x=3 \sigma^{2}\left(\sigma^{2}=1\right)$ and the data shows the maximum amplitude of noise is close to 3. Therefore, Q_MAX should be taken as 4 because the modulated amplitude of signal is 1.

Step2: estimate the quantized interval value " $\triangle$ ". There is no strict criterion to calculate as long as the appropriate value. For instance, assuming that $\left|\mathrm{Q} \_\mathrm{MAX}\right|=4, \triangle$ would get the proper values for 0.1 to 0.4 . It can be summarized as follows: the quantized grid is denser and the performance of demodulation is better for smaller $\triangle$-value; the quantized grid is sparser and the latency is shorter for bigger $\triangle$-value.

Step3: quantized constellation is drawn in Fig.2. As shown, take each central point of the quantized area as its quantification point and put all these points of every quantification interval as the input values into traditional demodulation algorithm such as Log-Map. The number of quantification points is

$$
\left(\frac{2 \times Q_{-} M A X}{\Delta}\right)^{2}
$$

Thus, we can get all bit soft values (i.e., bit log-likelihood ratio) corresponding to this quantification area.

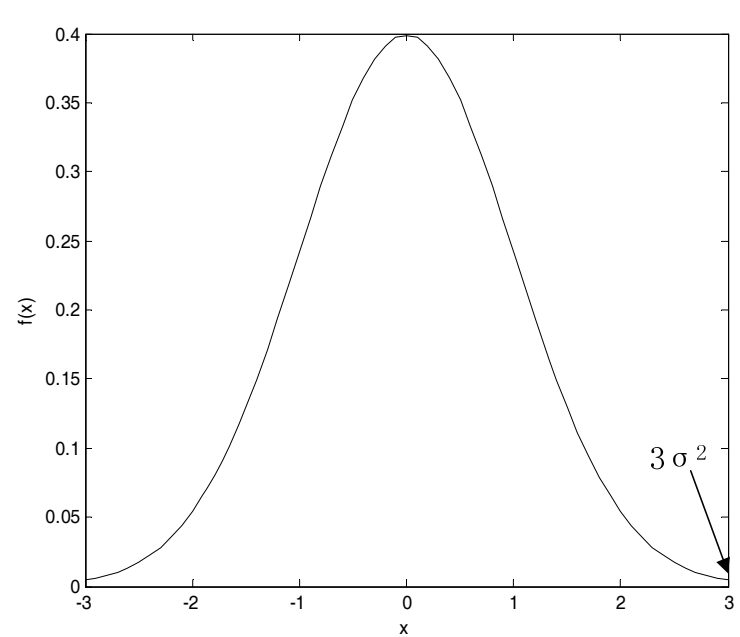

Fig.1. Probability density curve of the standard Gaussian distribution.

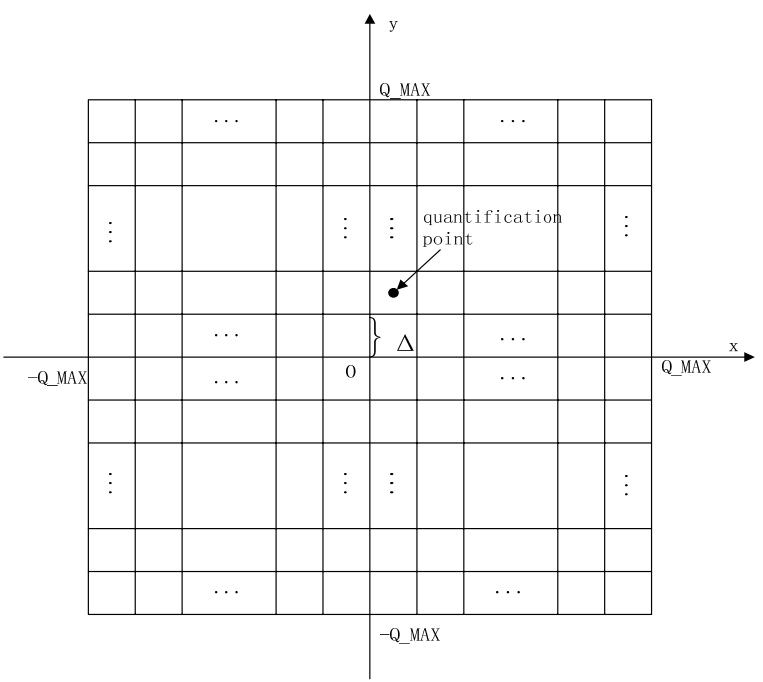

Fig.2. The quantized constellation diagram.

Step4: tabulation. Make a lookup table with values of every quantification point and its corresponding bit log-likelihood ratio. This table is ready for demodulation queries. 
The above is the pretreatment part. Making a lookup table doesn't take up the communication time because this process proceeds before regular communications.

Quantizing received symbols. This part is to quantify the received symbols. Fig.3 shows a quantification interval in a magnified form and it makes clear that symbols which are mapped to this interval are all quantified as its center point (i.e., the quantification point).

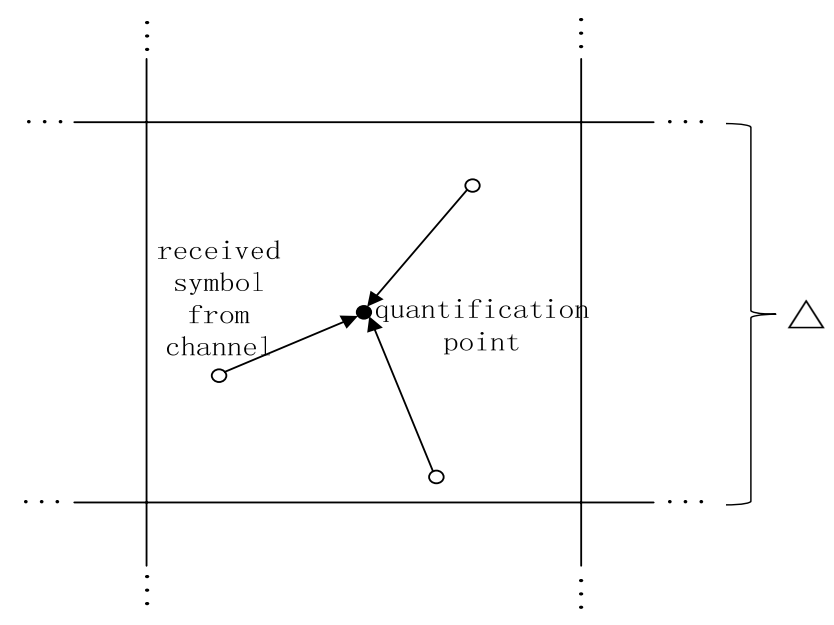

Fig.3. The quantification interval in a magnified form.

The concrete quantification steps are as follows:

Step1: the modulated symbol is a complex value which can be expressed as

$s=s_{I}+j s_{Q}$

where $s_{I}$ and $s_{Q}$ respectively represent the value of $X$ coordinate and $Y$ coordinate in the constellation. We can judge which quantification interval the symbol is mapped in according to the values of $X$ and $Y$.

Step2: take the centre point as quantification point of the received symbol.

The above is the quantizing received symbol part. Thus, we get the discrete value of received symbol rather than the continuous value.

Bit value search. When the demodulation end receives a symbol and quantifies it, system can query the bit soft values corresponding to this quantized point against the lookup table made in part $A$.

The traditional demodulation structure and proposed demodulation structure are shown in Fig.4.

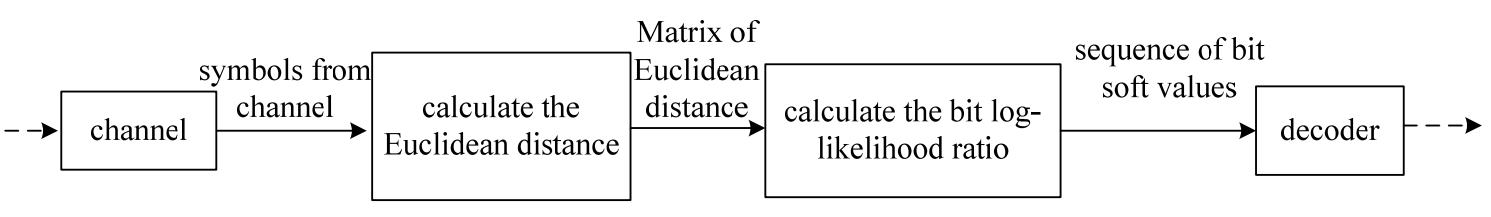

(a)

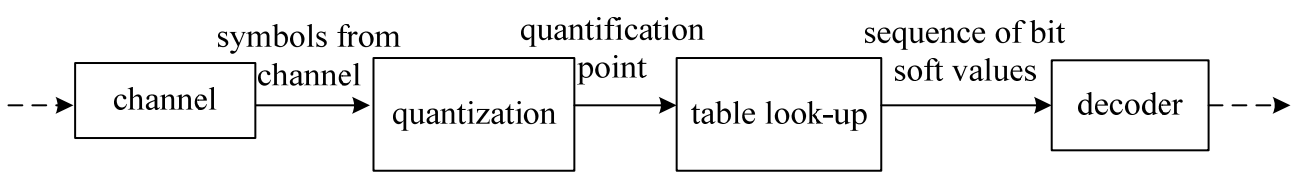

(b)

Fig.4. Demodulation structure. (a) traditional demodulation structure, and (b) proposed demodulation structure.

The two steps of traditional demodulation both require a great deal of computation. However, the proposed demodulation scheme doesn't spend time to calculate in the process of communication, and only takes a very little of time on checking the table. 


\section{Simulation Results}

A frame of 1024 bits is considered and at least 70 error frames are encountered. The encoder is convolutional code with generator polynomials (feedback, redundancy) $(13,15)_{\text {oct }}$, and the code rate is $R=1 / 2$. The modulation and demodulation adopt Quad-Phase Shift Keying (QPSK) with Gray mapping. The demodulation algorithm in pretreatment employs Log-Map. Simulations are executed under the condition of AWGN channel.

The statistical analysis and channel estimation are needed in order to set the Q_MAX value in the pretreatment stage. Fig.5 shows the noise amplitude distribution when the estimate of signal-tonoise ratio (SNR) is $0.5 \mathrm{~dB}$. We can consider the amplitude distribution as the probability density distribution. $\left|\mathrm{Q} \_\mathrm{MAX}\right|$ is less than 3 from the distribution and $\left|\mathrm{Q} \_\mathrm{MAX}\right|$ is extended to 4 to ensure that there is a low-probability event. The final value of $\left|\mathrm{Q} \_\mathrm{MAX}\right|$ is 5 because the extended value 4 need add the modulated amplitude of signal 1. Fig.6 shows the bit error rate (BER) comparisons about traditional demodulation and quantification demodulation with different $\triangle$ values $(\triangle=0.1$, $0.2,0.3$ and 0.4 ).

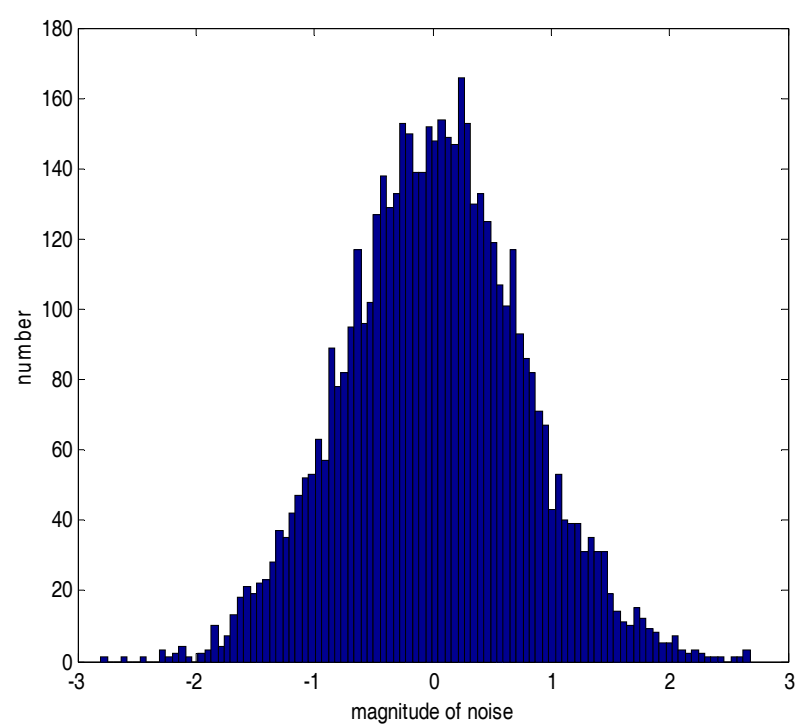

Fig.5. The noise amplitude distribution.

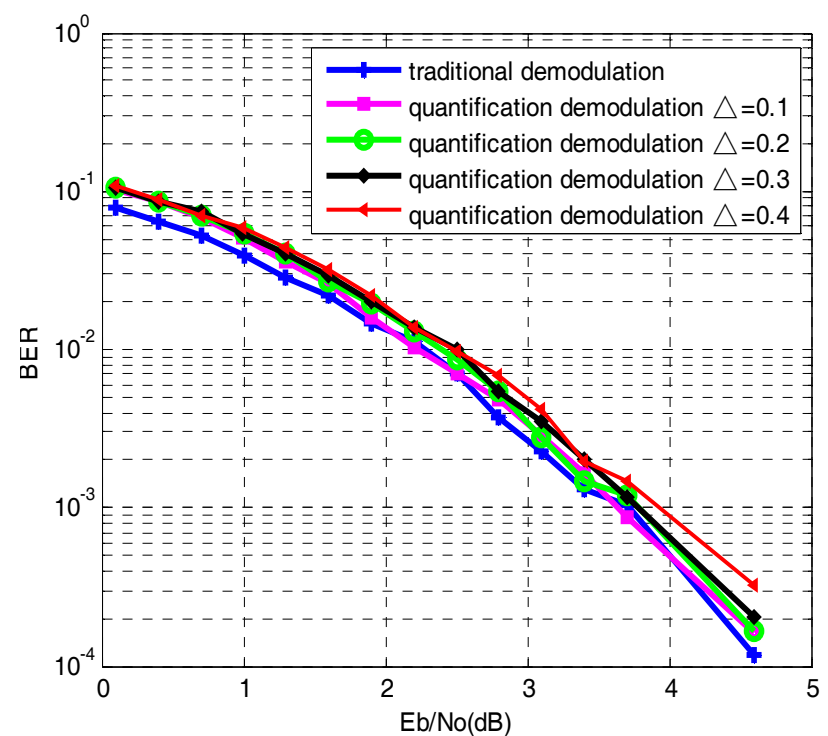

Fig.6. BER against SNR.

Obviously, performance of quantification demodulation is better than the other $\triangle$ values when $\triangle=0.1$ and almost the same as traditional demodulation when $\mathrm{SNR}>1.5 \mathrm{~dB}$. Performance gain is decreased by about $0.1 \mathrm{~dB}$ when $\triangle=0.2$ relative to $\triangle=0.1$. Although decoding performance decreases slightly with the increasing of $\triangle$, the delay is getting shorter. Table 1 lists the latency (time) comparisons about traditional demodulation and quantification modulation with different $\triangle$ values.

Table 1. Latency comparisons about two demodulation structures, $\left|\mathrm{Q} \_\mathrm{MAX}\right|=5$, demodulation algorithm adopts Log-Map

\begin{tabular}{|c|c|c|c|}
\hline demodulation structure & parameter1 & parameter2 & latency(s) \\
\hline traditional demodulation structure & & & 98 \\
\hline \multirow{7}{*}{ proposed demodulation structure } & $\triangle$ value & $\begin{array}{l}\text { quantification } \\
\text { interval } \\
\text { number }\end{array}$ & $\begin{array}{l}\text { latency(s) (percent-age } \\
\text { reduction) }\end{array}$ \\
\hline & 0.1 & 10404 & $27(72.4 \%)$ \\
\hline & 0.15 & 4624 & $17(82.7 \%)$ \\
\hline & 0.2 & 2704 & $16(83.7 \%)$ \\
\hline & 0.25 & 1764 & $10(89.8 \%)$ \\
\hline & 0.3 & 1296 & $8(91.8 \%)$ \\
\hline & 0.4 & 676 & $6(93.9 \%)$ \\
\hline
\end{tabular}


Table 1 indicates that quantification demodulation can take on much less delay. As can be seen from the table, if $\triangle$ increases linearly, reduced latency is not linear.

\section{Conclusions}

The amplitude of modulated digital signal is discrete and it will become continuous after being transferred through a noisy channel. This paper proposes a new demodulation structure by quantizing the constellation and using a lookup table in BICM system. The novel structure quantifies these continuous noisy signals as discrete signals. Then, it is convenient and quick to look up the bit soft values against a lookup table. Different from the traditional demodulation structure completely, the proposed method will omit the large number of the calculations for demodulation. The boundary of the quantized area $\left|\mathrm{Q} \_\mathrm{MAX}\right|$ and quantized interval value " $\triangle$ " will influence the latency and decoding performance. Simulation results confirm that the novel structure can reduce the delay by $72.4 \%$ to $93.9 \%$ when the quantized interval is from 0.1 to 0.4 . Meanwhile, the performance gain only reduces about $0.1 \mathrm{~dB}$.

\section{Reference}

[1] G. Ungerboeck, "Channel coding with multilevel/phase signals," IEEE Trans. Inform. Theory, vol. IT-28, pp. 56-67, Jan. 1982.

[2] E. Zehavi, "8-PSK trellis codes for a Rayleigh channel," IEEE Trans. Commun., vol. 40, no. 5, pp. 873-884, May 1992.

[3] Kenarsari-Anhari, A., Lampe, L., "Performance Analysis for BICM Transmission over Gaussian Mixture Noise Fading Channels," IEEE Trans. Commun., vol. 58, no. 7, pp. 19621972, July 2010.

[4] Xu, W., Gu, C., Li, C., Sarrafzadeh, M., "Robust doppler radar demodulation via compressed sensing,” IEE Electronics Letters., vol. 48, no. 22, pp. 1428-1430, Oct. 2012.

[5] Gong, C.-S.A., "Investigation of efficient ASK demodulation for wirelessly powered niodevices,” IEE Electronics Letters., vol. 48, no. 4, pp. 203-204, Feb. 2012.

[6] L.R. Bahl, J. Cocke, F. Jelinek, and J. Raviv, "Optimal decoding of codes for minimizing symbol error rate,” IEEE Trans. Information Theory, vol. IT-20, pp. 284-287, Mar. 1974.

[7] Robertson, P., Villebrun, E., and Hoeher, P., "A comparison of optimal and sub-optimal MAP decoding algorithms operating in the log domain," Proc. IEEE Int. Conf. on Communications, (ICC'95), Seattle, WA, USA, June 1995, pp. 1009-1013.

[8] C. Berrou, P. Adde, E. Angui, and S. Faudeil, "A low complexity softoutput viterbi decoder architecture," in Proc. of IEEE Intl. Conf. on Com., Geneva, Switzerland, pp. 737-740, 1993. 